\title{
Les frontières de la violence au Kenya
}

29 décembre 2007 - 29 février 2008

\section{Claire Médard}

\section{(2) OpenEdition}

\section{Journals}

Édition électronique

URL : http://journals.openedition.org/echogeo/2335

DOI : 10.4000/echogeo.2335

ISSN : 1963-1197

\section{Éditeur}

Pôle de recherche pour l'organisation et la diffusion de l'information géographique (CNRS UMR 8586)

Référence électronique

Claire Médard, "Les frontières de la violence au Kenya », EchoGéo [En ligne], Sur le Vif, mis en ligne le 12 mars 2008, consulté le 02 mai 2019. URL : http://journals.openedition.org/echogeo/2335 ; DOI : 10.4000/echogeo.2335

Ce document a été généré automatiquement le 2 mai 2019.

\section{(c) (i) (9)}

EchoGéo est mis à disposition selon les termes de la licence Creative Commons Attribution - Pas d'Utilisation Commerciale - Pas de Modification 4.0 International 


\section{Les frontières de la violence au}

\section{Kenya}

29 décembre 2007 - 29 février 2008

\section{Claire Médard}

1 En 2007, la suspicion de fraude, à présent avérée, qui permet au président Mwai Kibaki, à la tête du Party of National Unity (PNU), de rester au pouvoir, enflamme, dès le 29 décembre au soir, les circonscriptions contrôlées par l'opposition de l'Orange Democratic Movement (ODM). Le soutien à l'ODM est important dans tout l'ouest du pays : en pays luo, autour de la ville de Kisumu, région d'origine du dirigeant du mouvement, Raila Odinga, en pays kalenjin, fief du numéro deux du mouvement, Daniel Ruto, représentant de la ville d'Eldoret, et en pays luhya. Le président Kibaki tire son soutien de la province du centre, du pays kikuyu. Dans toutes les villes de l'ouest du Kenya, y compris dans les plus petits villages, les Kikuyu, établis de longue date, sont forcés de trouver refuge dans des lieux sûrs. C'est à Eldoret que se déroule l'épisode marquant de l'incendie d'une église qui provoque la mort des personnes qui s'y sont réfugiées, le $1^{\text {er }}$ janvier 2008. La violence de miliciens kalenjin trouve ses racines dans les mobilisations politiques de la décennie précédente et renvoie à la 'promotion' d'une ethnicité kalenjin par le régime du président Moi. 
Figure 1 - Principaux groupes ethniques

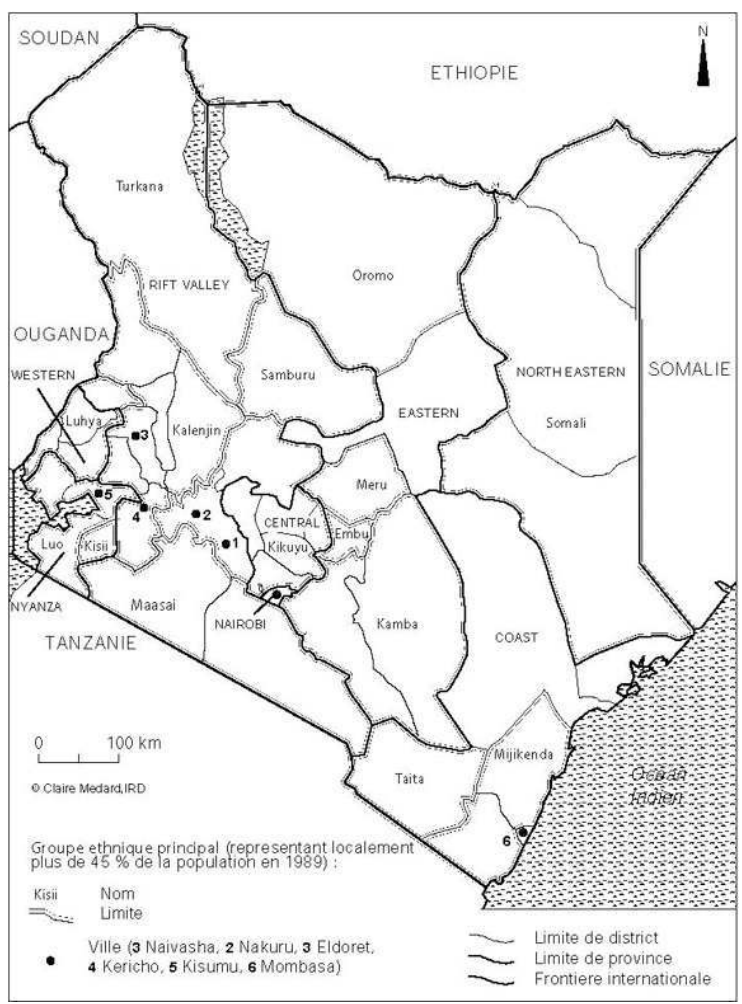

2 A partir de ces élections truquées, la violence se déchaîne et fait la une de la presse internationale. Deux mois plus tard, les morts ont passé le seuil du millier (1 500 d'après la police) et les déplacés internes sont évalués à 300000 par la Croix-Rouge. A titre de comparaison, durant les années 1990, au cours de plusieurs épisodes de violence électorale, les déplacés internes au Kenya sont estimés à 300000 sur toute la période. Pour évoquer brièvement ce conflit, je rappellerai les évènements récents et les dynamiques territoriales actuelles.

Ancrages d'une violence multiforme

3 Les violences récentes ne sont pas sans rappeler les conflits des années 1990. A l'époque, ces conflits se rattachent à une stratégie du président Daniel Arap Moi (en exercice de 1978 à 2002) pour conserver le pouvoir. Son régime, se sentant menacé par l'avancée démocratique dans le pays, préconise la préférence régionale (idéologie majimbo), pour le bénéfice de "minorités », comme alternative au multipartisme. Une vague de violence organisée ravage le Kenya, aboutissant localement à la constitution de fiefs électoraux et ethniques. L'ancien domaine foncier européen, africanisé depuis l'indépendance, constitue des terres convoitées par les partisans du régime Moi. S’ils parviennent à l'époque à chasser les populations non kalenjin d'une région située entre Nakuru, Eldoret et Kericho, le cadre légal de la propriété foncière en freine la réappropriation.

Du 29 décembre 2007 au 10 janvier 2008, durant le mouvement de protestation initial, des manifestations de violence partent des principales villes du Kenya avant d'atteindre les campagnes. La ville de Kisumu est dévastée. Les violences se déchaînent dans le quartier de Kibera à Nairobi, encerclé par les forces de l'ordre. Les régions rurales les plus touchées se situent dans les fiefs électoraux kalenjin. Dans le triangle situé entre Nakuru, Eldoret et Kericho, dans cette région d'où les Kikuyu ont été chassés, durant les années 
1990, un processus de recolonisation agricole par de nouveaux migrants kikuyu, s'était accéléré avec le régime du président Kibaki. Déjà, durant la campagne électorale, les tensions étaient palpables ${ }^{1}$. Après la réélection frauduleuse de Mwai Kibaki, les milices kalenjin mettent le feu aux habitations kikuyu. Partout, la violence touche les Kikuyu, mais également d'autres populations parmi lesquelles des Luhya et des Kisii accusés localement de ne pas avoir soutenu l'ODM. Durant cette vague de violence, la circulation sur les axes principaux qui traversent le Kenya est à l'arrêt, d'où la mobilisation du président de l'Ouganda voisin, Museveni, dès les premiers jours de la crise, pour trouver une solution. Des barrages sont érigés par des partisans de l'ODM qui organisent le lynchage de Kikuyu. Une réaction immédiate à toutes ces violences, dont les Kikuyu sont la cible, se produit à quelques centaines de kilomètres de distance dans la capitale Nairobi et dans la province du Centre, d'où les Luo et les Luhya sont chassés. Des milices d'autodéfense proclament que pour tout Kikuyu tué, dix Luo seront exécutés. Le retour au calme, à Kisumu, se fait également dans la violence avec l'envoi des forces de l'ordre qui tirent par balle. Ce bref aperçu donne la mesure de l'enracinement local de ces conflits par rapport à des enjeux politiques et fonciers.

5 Cette première phase du conflit est suivie d'un répit. Après une accalmie de deux semaines environ, de nouvelles violences se produisent. Cette fois-ci, des miliciens kikuyu reprennent l'initiative. La violence part de Nakuru, où de nombreux réfugiés se retrouvent, et s'étend, en direction du sud-est, vers Naivasha. A Nakuru, dans la ville même, des gangs kikuyu s'en prennent aux Kalenjin. Cette attaque est suivie par une contre attaque de milices kalenjin contre des paysans kikuyu établis dans une région située au nord de la ville ${ }^{2}$. La croisade menée par des miliciens kikuyu atteint Naivasha le 27 janvier 2008, où le meurtre de dix personnes réfugiées dans une église fait écho à l'attaque de l'église d'Eldoret, un mois plus tôt. Des barrages routiers sont érigés par des miliciens qui s'en prennent tout particulièrement aux Luo. En raison de l'importance de l'activité horticole, sur les rives du lac, le peuplement de la région de Naivasha, en forte croissance, était, avant la crise, cosmopolite. Les violences ont eu un impact direct sur l'activité productive en provoquant l'exil de la main d'œuvre luo et luhya très présente sur ces fermes. Des voix kikuyu se sont élevées pour revendiquer les emplois localement. Par ailleurs, dans la capitale Nairobi, des actes de violences autour d'évictions forcées se sont intensifiés dans certains quartiers, prenant tout le monde au dépourvu: les bâtiments sont parfois brûlés ou réquisitionnés sans autre forme de procès et les titres de propriété exigés par des miliciens. Une ségrégation spatiale sur le mode de quartiers luo ou luhya et de quartiers kikuyu s'est opérée.

6 Cette deuxième flambée de violence, début février 2008, marque le commencement d'une période d'expectative autour de la médiation pour la paix de Kofi Annan, ancien secrétaire générale des Nations Unis, qui cherche à rendre possible un accord entre les deux camps rivaux pour le partage du pouvoir. Tous les espoirs se fondent sur le succès de ces négociations malgré des attentes contradictoires et même des rumeurs inquiétantes concernant l'armement de factions rivales. Finalement, le 29 février 2008, un accord a été signé, avec la création d'un poste de premier ministre, destiné à Raila Odinga.

7 L'épisode récent de violence au Kenya se caractérise par sa brutalité. Les conflits des années 1990 avaient touché pour l'essentiel des populations rurales et avaient provoqué un accroissement de la population des villes. A l'heure actuelle, la dimension urbaine du conflit a pris de l'envergure. La propagande et le soutien du régime Moi à l'activisme électoral et ethnique, dans les années 1990, constituent un élément décisif pour expliquer 
la violence actuelle. L'alternance politique, depuis 2002, malgré les discours, n'a pas pris la réelle mesure de la crise de légitimité de l'Etat et n'a pas su le réformer en profondeur. La dimension locale de chaque conflit confère à la crise des ancrages spécifiques et une autonomie potentielle.

Négociations autour d'un héritage territorial

La dimension territoriale du conflit mérite d'être examinée dans une perspective historique. L'idée de territoires ethniques exclusifs qui se traduit, aujourd'hui, par le concept de préférence régionale ou de majimbo, n'est pas nouvelle au Kenya. Elle renvoie au cadre administratif du pays, hérité d'une colonisation de peuplement. Même si les colons européens n'étaient pas nombreux, ils se sont taillés un immense domaine foncier, allant principalement du Mont Elgon jusqu'à Nairobi. Dans la capitale, Nairobi, conçue comme européenne : les quartiers africains historiques sont circonscris territorialement. A l'échelle du Kenya, la ségrégation territoriale a eu un impact durable: difficile de comprendre son organisation spatiale, aujourd'hui, sans y faire référence. Durant la colonisation, les populations africaines sont rattachées administrativement à des réserves rurales et à des localités bien précises dans les villes, où elles sont censées résider. Au besoin, les populations migrantes sont renvoyées dans leur région rurale d'origine. Un système de permis (kipande) pour se déplacer et travailler en dehors de sa région d'origine, est mis en place. Par commodité administrative, le domaine africain est à son tour divisé territorialement en réserves ethniques : réserve kikuyu au nord de Nairobi, la réserve maasai au sud, la réserve luo en bordure du lac Victoria et la réserve luhya au sud du Mont Elgon, etc. A la différence des groupes cités précédemment, les sous-groupes Kalenjin, situés de part et d'autre du domaine foncier européen, ne sont pas unis territorialement durant la colonisation, ils ont des territoires administratifs distincts (réserve nandi, réserve kipsigis, pour ne citer que quelques exemples).

9 L'idée d'une appartenance territoriale exclusive, fondée sur un cadre territorial rigide, défini par des frontières linéaires, constitue une mutation territoriale apparue au cours du siècle dernier. Les réserves ethniques, instaurées par la colonisation, n'ont plus grandchose à voir avec les territoires antérieurs, assez flexibles, de ces sociétés où fronts pionniers et no man's land sont au cœur des dynamiques territoriales. L'intervention coloniale a contribué à forger de nouvelles ethnicités en leur conférant un fondement territorial rénové et une forme de permanence. L'idée d'homogénéité ethnique, suggérée par ces regroupements territoriaux, est à la fois nouvelle et, il convient de le souligner, illusoire. Elle s'oppose à des stratégies identitaires mouvantes et à des dynamiques migratoires mises en place depuis la colonisation dans un contexte d'accroissement démographique (Oucho, 2002). Le compartimentage ethnique traduit, certes, une appropriation plus serrée de l'espace, mais relève d'une toute autre logique, potentiellement déstabilisatrice. Par le principe qu'il introduit, l'appropriation d'une localité ne relève plus d'une occupation effective mais d'un droit territorial qui la dépasse. Parfois qualifié d' "ancestral », il s'apparente plutôt à un droit « colonial » sur un espace. Les frontières héritées de la colonisation ont été renégociées après l'indépendance, néanmoins leur logique territoriale qui transcende la localité demeure la même.

10 A l'indépendance, s'ouvre la perspective d'une reconquête territoriale (Droz, 1999). Jomo Kenyatta (1963-1978) affirme qu'il faut mettre fin au compartimentage ethnique et construire une nation. A la même époque, Daniel Arap Moi défend une position différente, plus proche de la conception coloniale, fondée sur une reconnaissance territoriale des 
communautés. Il défend ouvertement l'idée d'un partage territorial de la nation. Dans ce contexte, il cherche à promouvoir une ethnicité kalenjin englobante, dans laquelle différentes micros ethnicités sont fédérées autour d'une revendication portant sur le domaine foncier européen. Lorsqu'il est effectivement au pouvoir, durant les années 1990, les frontières d'anciennes réserves coloniales sont réactivées, de nouvelles apparaissent. A l'intérieur de la province de la Rift Valley, revendiquée territorialement par les Kalenjin, une nouvelle frontière marque de facto une coupure entre un domaine kalenjin, à l'ouest, et un domaine kikuyu, à l'est. Celle-ci se retrouve au cœur du conflit en 2007-2008. A l'échelle de la ville de Nairobi également, l'héritage colonial est instrumentalisé et de nouvelles frontières font leur apparition. La chasse aux Luo et aux Luhya s'organise dans certains quartiers populaires et en sens inverse d'autres sont épurés des Kikuyu Si certaines frontières trouvent leur origine directement dans l'Etat colonial, une ségrégation spatiale s'opère sous le coup de la peur et de la violence. Ces frontières internes et la violence associée semblent marquer la fin de l'illusion d'un Etatnation. Et pourtant, sur le plan des discours, on ne peut reprocher ni à Moi, ni à Kenyatta, ni à Kibaki de ne pas être nationalistes. En revanche, il convient de souligner que leur pratique du pouvoir et, en particulier, la manière dont l'Etat s'arroge le contrôle de l'accès à la terre, contredit leurs desseins nationalistes.

Conclusion : répercussions durables d'une crise politique

11 Si les positions de Kenyatta et de Moi sur la nation kényane sont contrastées, leur pratique du pouvoir les rapproche. Les deux régimes partagent des pratiques clientélistes d'accès aux ressources et une tradition de contrôle territorial. La crise actuelle, dans sa dimension ethnique, résulte de ces héritages politiques. Les violences ethniques et policières qui se font écho, aujourd'hui, risquent d'avoir des répercussions durables. Des frontières internes héritées de la colonisation ont été réactivées ou renégociées dans le cadre de ces conflits. Les retours dans les 'régions d'origine', sous le feu de la violence, ont prouvé en définitive à quelle point il s'agissait de catégories solides, héritées d'une pratique administrative. Lorsque la présence des populations locales est contestée sur un plan territorial, en référence à l'idée coloniale de réserve ethnique, remise à la sauce de stratégies politiques actuelles et qu'elles sont chassées, le calme peut revenir un temps. Jusqu'au jour où, dans l'engrenage de la violence, des revendications qui se fondent sur des mémoires concurrentes se répondent et finissent par inscrire les conflits dans la durée. Les territoires qui se sont façonnés dernièrement, dans la peur et la haine, peuvent paraitre nouveaux dans leur forme, mais pas dans leur logique. Ils marquent une étape nouvelle dans la construction de territoires et d'ethnicités sur le mode de l'exclusion. L'accord est parvenu pour l'instant à éviter une guerre déclarée, entre forces opposées, dont l'aboutissement logique serait une forme de balkanisation du Kenya.

\section{BIBLIOGRAPHIE}

Oucho, J, 2002. Undercurrents of Ethnic Conflict in Kenya. Leiden, Brill, African Social Studies Series $(235$ p.) 
Droz, Y, 1999, Migrations kikuyus. Des pratiques sociales à l'imaginaire. Neuchâtel, Editions de l'Institut d'ethnologie (462 p.)

\section{NOTES}

1. Communication personnelle, Hervé Maupeu, le 08/02/2008, Journée d'étude de la revue Politique Africaine.

2. Daily Nation, le 27 janvier 2008.

\section{RÉSUMÉS}

$\mathrm{Au}$ cours des violences post électorales récentes, au Kenya, de nombreux observateurs ont été surpris par la forme prise par les protestations partisanes. Elle nous rappelle que la fabrique territoriale de l'ethnicité est toujours d'actualité dans ce pays. La réactivation de frontières internes qui portent la marque des violences du passé, certaines héritées de la colonisation et d'autres renégociées depuis l'indépendance, ont mis en péril l'Etat.

During the recent post electoral crisis in Kenya, the turn of the protest took many by surprise. We were reminded that the making of ethnicity is on ongoing process in which violence plays a part. New boundaries have appeared, re-enacting old divisions and creating new rifts. Once the mark of administration, internal boundaries now threaten the existence of the State.

\section{INDEX}

Mots-clés : territoire de l'État, ethnicité, conflit, frontières internes

Keywords : State territory, ethnicity, conflict, internal boundaries

\section{AUTEUR}

\section{CLAIRE MÉDARD}

Claire Médard est chercheur à l'IRD. Elle a récemment publié : 1. Les conflits 'ethniques' au Kenya. Une question de votes ou de terres. Afrique contemporaine, 1996, $4^{\mathrm{e}}$ trimestre, $\mathrm{p}$. 62-74 2. La réforme de l'administration territoriale n'aura pas lieu au Kenya, L'Afrique orientale. Annuaire 2005. Paris. L'Harmattan, 2007, p. 3-10 3. Elus, miliciens et prophètes. Violences au Mont Elgon, Kenya, 2006-2008. Politique Africaine 2008, nº 109. 